\title{
Modelling of the access part of a multi-service mobile network with service priorities
}

\author{
Slawomir Hanczewski*, Maciej Stasiak and Piotr Zwierzykowski
}

\begin{abstract}
This paper presents a methodology for the modelling and optimization of multi-service radio access for universal mobile telecommunications system (UMTS) networks. The paper provides a description of the basis for modelling of a tandem pair: a wideband code division multiple access (WCDMA) interface and a lub interface. The models involved in the study take into consideration a possibility of setting priorities to a number of selected traffic classes. Particular attention is given to the development of simple computational algorithms that would make it possible to determine the blocking probability for call streams with different priorities. The results of the analytical calculations are then compared with the results of simulation experiments, which confirms the high accuracy of the proposed analytical solutions.
\end{abstract}

Keywords: UMTS network; WCDMA interface; lub interface; Multi-service traffic, priorities

\section{Introduction}

Modern mobile networks service mixtures of different traffic streams with different quality requirements. Both the traffic that requires appropriate quality parameters (e.g., VoIP traffic, speech) and the traffic with lower requirements (e.g., www traffic) are serviced in shared network resources. Given that there are different requirements that impose different quality demands for services and users of the network, manufacturers of network devices have started to introduce appropriate functions and traffic control mechanisms to adjust their products to meet these challenges.

Mobile network users are more and more willing to pay whatever it costs for access to links that offer a higher sustainable data throughput. From a network operator's perspective, the introduction of priorities for some selected groups of users is then very advantageous and profitable. A differentiation in the access to the network is required to be not only at the user level but also results from specific quality requirements of some services. This entails, in turn, the need for the introduction of appropriate quality of service (QoS) mechanisms in network devices. QoS mechanisms in the universal mobile

*Correspondence: slawomir.hanczewski@put.poznan.pl

Chair of Communication and Computer Networks, Poznan University of Technology, Polanka 3, 60-965 Poznań, Poland telecommunications system (UMTS) network are available, for example, in the radio interface that is responsible for the highest degree of limitation imposed on network capacity. Such mechanisms are also available in the Iub interface, located between NodeB and radio network controller (RNC) [1-4]. For the purpose 3G network analysis, dimensioning, and optimization, it is then necessary to develop appropriate analytical models of these interfaces.

The literature of the subject abounds in the presentations of models of interfaces of mobile systems that give priority to a selected group of services [5-8] or to users $[9,10]$. These models are essentially based on the model of the multi-service full-availability group [11, 12]. In this paper, however, to model the wideband code division multiple access (WCDMA) interface, a model of a multi-service Erlang's ideal grading is used [13, 14]. In addition, the paper proposes a model of the non-fullavailability group with priorities based on the approach that has been earlier adopted for the analysis of priorities in the full-availability group $[5,6]$.

The non-full-availability group is the so-called statedependent system $[14,15]$. This dependence results from the particular structure of the system in which calls arriving at the input have access to a limited amount of resources, substantially lower than the total capacity of

\section{是 Springer}


the system. Until the 1980s, non-full-availability groups with single-service traffic were used in electromechanical switching nodes of telecommunications networks. With the advent of electronic nodes, groups of this type ceased to be used in their direct form, though they were, and still are, used in analytical models of more complex systems, such as, for example, single- and multiservice switching networks [16-18], network systems with reservation $[19,20]$, systems with traffic overflow [21-23], video on demand (VoD) systems [24, 25], or radio and Iub interfaces of the UMTS system [26-28]. One of the most extensive elaborations on non-full-availability groups is provided in the [29]. This paper presents all of the most important methods of modelling single-service non-full-availability groups. Among different types of the non-full-availability group, particular attention should be given to Erlang's ideal grading, the structure of which and the first single-service analytical model was presented by Erlang [30]. The first model of the ideal grading with multi-service traffic and identical value of the availability parameter for all classes of calls serviced by the group is proposed in [13]. Stasiak and Hanczewski [31] discuss a model of the group with multi-service traffic and a variable value of the availability parameter. This model is then expanded to include the possibility of performing calculations for non-integer vales of availability [31]. The most extensive description of multi-service non-full-availability groups is provided in [14] in which different models of multi-service ideal non-full-availability groups, defined for different types of call streams and different structures of groups, are presented.

The idea of the non-full-availability group makes it possible to take into consideration the influence of interference from other cells in a given cell, since an increase in interference directly leads to a decrease in the availability of a given group. The relationship between the changes in availability and changes in the level of interference is discussed in [28, 32].

So far, the literature on the subject has been addressing exclusively models of single interfaces with priorities. Systems in which priorities have been simultaneously introduced to two interfaces, e.g., the radio interface and the Iub interface, have not been analyzed yet. This paper proposes a model of a system with priorities that enables the analysis of such systems to be performed. The mutual dependence between traffic serviced in both interfaces is taken into account by the application of the fixed-point methodology $[33,34]$ that makes it possible to analyze the call an analysis of the call service in a number of systems simultaneously.

The paper is an overview of the subject in ten sections. Section 2 presents the architecture of the access part of the UMTS network. Sections 3 and 4 provide a description of the traffic representation in the Iub and the WCDMA interfaces. Sections 5 and 6 discuss analytical models of the Iub and the WCDMA interface, while Section 7 includes a description of a model of the system with priorities. Section 8 presents a model for setting up connections in a system that consists of a cascade of WCDMA and Iub interfaces. The following section, Section 9, provides an example illustrating the available possibilities of the application of the model in analyzing the UMTS system. Finally, the conclusions and considerations which emerge from the study and the results of the experiments are provided in the last section of the paper.

\section{UMTS network architecture}

Figure 1 shows the access part of the UMTS network. It is composed of users added through a radio interface to multiple base stations (NodeB). The base stations are, in turn, connected through the Iub interface to the controllers of base stations (RNC). The important thing is that the number of base stations connected (added) to one RNC depends on the location and producer of the applied devices.

A number of sectors can be run within one base station (usually from 3 to 6), whereas the number of cells that can operate within one sector can be as many as the carriers of a given operator (e.g., R99, high speed packet access (HSPA) 1-carrier, HSPA dual-carrier, and UMTS900). Hence, if we assume that we consider only one carrier, then the number of cells added to a given base station ranges from 3 to 6 . The number of cells that can be added to a single RNC actually depends on the producer and the type of license held by the operator. In the main, no more than 5000 cells can be added to one RNC, which means that one can get from about 800 to about 1600 base stations for three or six sectors, respectively.

It should be stressed, however, that both the number of sectors in a base station and the number of cells added to RNC can substantially differ depending on the solutions preferred by given producers of network devices for UMTS. In the UMTS network, priorities can be assigned to particular services. Priorities define the sequence of resource allocation. This sequence may result, in the case of services with lower priority, in a decrease in the amount of resources or may even enforce a termination of a service (in an instance when there are no sufficient resources to be allocated to services with higher priority). The decision of whether a priority is allocated or not is made by the network operator who defines its importance (significance) in the core network. Hence, the call service process of calls with different priorities is carried out both in the radio part as well as in the resources of Iub interfaces of the access part of the UMTS network. The execution of prioritization means that the traffic management mechanism implemented at the call admission control (CAC) and/or call congestion control (CCC) levels, which are usually 


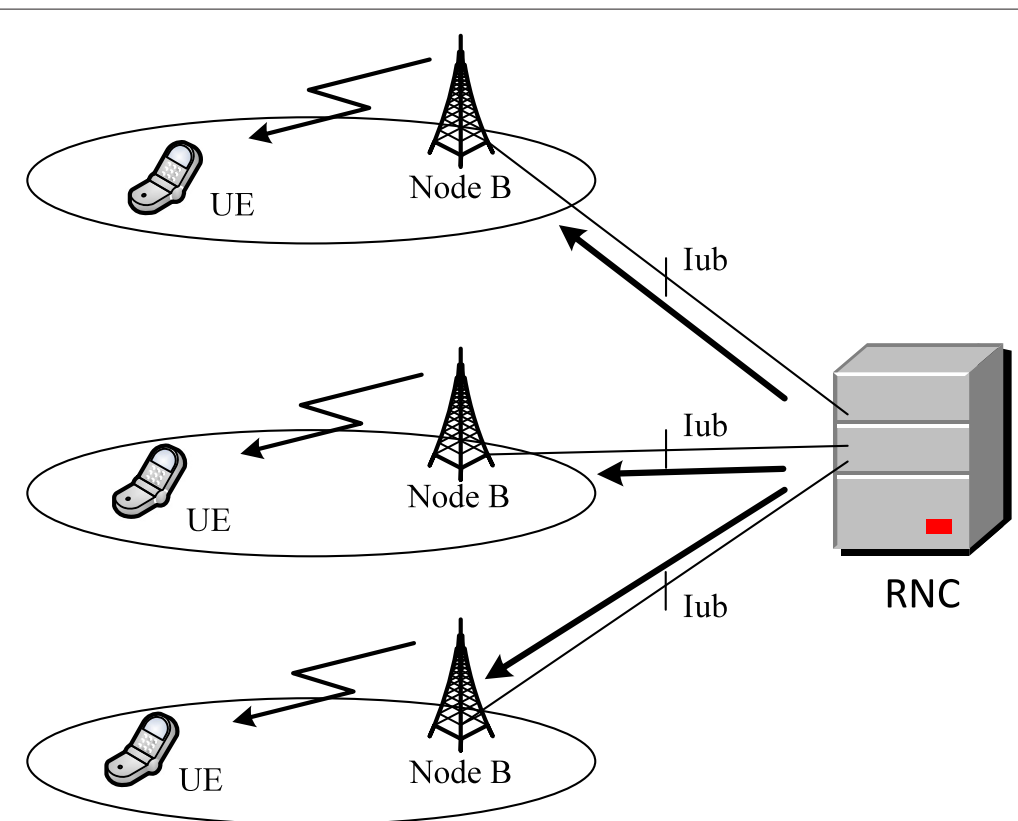

Fig. 1 Architecture of the access part of the UMTS network (user equipment (UE))

executed in the RNC software, is being used. In practice, many operators execute the prioritization of services in such a way as to make it imperceptible to users, while the priorities assigned to different categories of users are visible. Generally, the available categories of users are labelled as platinum, gold, silver, and bronze, while each of the groups included is assigned appropriate service parameters, e.g., through the allocation of specific priorities $[9,10]$. The process of defining priorities is executed at both the radio interface level and the Iub resource level.

\section{Traffic representation in the lub interface}

Let us assume that an Iub interface is offered a mixture of different packet streams. Analytical traffic models are constructed based on the internal structure of the packet stream or the so-called call stream in which a packet stream with a variable bit rate is treated as a single call that is characterized by a constant bit rate and appropriate service time. The term "call" is then meant to be understood as a packet stream or its section, which is related to a given service $[2,35]$. It has been proved, on the basis of simulation experiments and measurements carried out in networks, that thus defined calls can be described by the Poisson streams characteristics $[2,35,36]$ in which variable bit rates of packet streams are replaced by constant bit rates called the equivalent bandwidth [37]. The equivalent bandwidth is determined depending on and in relation to the capacity of the system, the maximum and the average bit rate of the packet stream, the variance of the bit rate, packet delay, and other important and relevant parameters for a given service [38, 39].
Let us assume that the total bit rate of the Iub interface is $C$. It is adopted in this paper that a packet stream of a given call of class $i$ that is offered to the Iub interface will be represented by a constant bit rate $\left[c_{i}\right]_{M}$ a (that corresponds to the equivalent bandwidth). Such an approach makes it possible to perform the so-called discretization of the system [38, 39], i.e., the expression of the capacity of the system, as well as the demands of individual traffic classes, in the same units, called allocation units (AU). The allocation unit $c_{\mathrm{AU}}$ defines the minimum bit rate in such a way that the bit rates of all calls are its multiple numbers. The allocation unit can be thus defined as the greatest common divisor (GCD) of all equivalent bandwidths in a given system $[38,39]$ :

$$
c_{\mathrm{AU}}=\operatorname{GCD}\left(\left[c_{1}\right]_{M}, \ldots,\left[c_{i}\right]_{M}, \ldots,\left[c_{M}\right]_{M}\right),
$$

where $M$ is the number of call streams offered to the system.

After determining the $c_{\mathrm{AU}}$ parameter, both the demands of individual call classes $\left[t_{i}\right]_{M}$ (i.e., the number of allocation units necessary to set up a call of class $i$ ) and the capacity of the system $V$ can be expressed in AUs:

$$
\begin{aligned}
& {\left[t_{i}\right]_{M}=\left\lceil\frac{\left[c_{i}\right]_{M}}{c_{\mathrm{AU}}}\right\rceil,} \\
& V=\left\lfloor\frac{C}{c_{\mathrm{AU}}}\right\rfloor .
\end{aligned}
$$

Assuming that the traffic stream expressed by the call stream has the Erlang traffic characteristics, the average 
intensity $\left[A_{i}\right]_{M}$ of traffic of class $i$ offered to the system can be expressed by the following formula:

$$
\left[A_{i}\right]_{M}=\frac{\left[\lambda_{i}\right]_{M}}{\left[\mu_{i}\right]_{M}}
$$

where $\left[\lambda_{i}\right]_{M}$ is the intensity of a Poisson call stream of class $i(1 \leq i \leq M)$ and $\left[\mu_{i}\right]_{M}$ is the average service intensity for call of class $i$.

\section{Traffic representation in the WCDMA radio interface}

In any analysis of radio interfaces of mobile networks, it is more convenient to use the notion of noise load $\left[L_{i}\right]_{M}$, introduced by a call of class $i$, instead of bit rates. It is a non-dimensional parameter and defines a fraction of the occupancy of the radio interface by a call of class $i$. The non-linear dependence between the noise load $\left[L_{i}\right]_{M}$ and the equivalent bandwidth with a bit rate $c_{i}$ is expressed by the following formula [40]:

$$
\left[L_{i}\right]_{M}=\frac{1}{1+\frac{W}{\frac{E_{b}}{N_{0}}}\left[c_{i}\right]_{M}\left[v_{i}\right]_{M}},
$$

where:

- $W$ is the chip rate of the dispersing signal that defines the velocity at which the input signal is dispersed,

- $\left[v_{i}\right]_{M}$ is the activity coefficient that defines the percentage occupancy of a transmission channel occupied by a user of class $i$, and

- $E_{b} / N_{0}$ is the ratio between the energy per one bit $E_{b}$ and the noise spectral density $N_{0}$.

This paper assumes that the packet stream of a given call of class $i$ will be represented by a constant noise load $\left[L_{i}\right]_{M}$, which would correspond to the bit rate of a equivalent bandwidth $c_{i}$. In such a case, AU for the radio interface will determine a certain minimum noise load $L_{\mathrm{AU}}$, such that the noise loads of individual calls are its multiple numbers. The allocation unit can then be defined as the GCD of all noise loads in a given system [38, 39]:

$$
L_{\mathrm{AU}}=\mathrm{GCD}\left(\left[L_{1}\right]_{M}, \ldots,\left[L_{i}\right]_{M}, \ldots,\left[L_{M}\right]_{M}\right),
$$

where $M$ is the number of call streams offered to the system.

After determining the parameter $L_{\mathrm{AU}}$, both the demands of individual call classes $\left[t_{i}\right]_{M}$ (i.e., the number of allocation units necessary to set up a call of class $i$ ) and the capacity of the radio interface $V$ can be expressed in AUs:

$$
\begin{aligned}
& {\left[t_{i}\right]_{M}=\left\lceil\frac{\left[L_{i}\right]_{M}}{L_{\mathrm{AU}}}\right\rceil,} \\
& V=\left\lfloor\frac{1}{L_{\mathrm{AU}}}\right\rfloor,
\end{aligned}
$$

where value 1 defines the theoretical capacity of an isolated radio interface for the uplink or the downlink.

The intensity of the offered traffic of a given class does not depend on the type of the interface and can be expressed by Formula 4. Observe that due to the nonlinear dependence between the noise load and bit rate (Eq. 5), the number of allocation units necessary for a connection of a given class to be set up will be different in the radio interface and in the Iub interface.

\section{Model of the lub interface}

To model the occupancy distribution in the Iub interface a multi-service model of the so-called full availability group (FAG) can be used. Let us consider a FAG to which a mixture of $M$ call streams with different bit rates is offered. This group is defined by the demands of individual call classes $\left[t_{i}\right]_{M}(1 \leq i \leq M)$ expressed in AUs, the capacity $V$, also expressed in AUs, and traffic intensity $\left[A_{i}\right]_{M}$ $(1 \leq i \leq M)$ of individual call classes, expressed in Erlangs. In the case of the Iub interface, these parameters are defined by Eqs. 2-4. The occupancy distribution in FAG can be determined recursively on the basis of the following formula $[11,12]$ :

$$
n\left[P_{n}\right]_{V}=\sum_{i=1}^{M}\left[A_{i}\right]_{M}\left[t_{i}\right]_{M}\left[P_{n-t_{i}}\right]_{V},
$$

where $\left[P_{n}\right]_{V}$ determines the occupancy probability of $n$ AUs in the Iub interface $(0 \leq n \leq V)$.

The blocking probability $\left[E_{i}\right]_{M}$ for traffic of class $i$ is determined by the sum of blocking states, i.e., the states in which there is no sufficient number of AUs to set up a connection of class $i$ :

$$
\left[E_{i}\right]_{M}=\sum_{n=V-\left[t_{i}\right]_{M}+1}^{V}\left[P_{n}\right]_{V} .
$$

Formulas 9-10 have been derived on the basis of the analysis of the Markovian process taking place in FAG $[11,12]$. If the system services only one call stream, then Formula 10 can be reduced in its essence to the so-called Erlang B formula.

Further on in our considerations, the blocking probability for each traffic class, obtained on the basis of Models 9-10, will be presented, for convenience, in the form of the following functional dependence:

$$
\mathbf{E}_{\text {lub }}=\left\{\left[E_{i}\right]_{M}: 1 \leq i \leq M\right\}=\operatorname{FAG}(\mathbf{A}, \mathbf{t}, V, M),
$$

where $\left[E_{i}\right]_{M}$ is defined by (10), and $\mathbf{t}$ and $\mathbf{A}$ are sets of demands and traffic intensities of individual call classes:

$$
\begin{aligned}
& \mathbf{t}=\left\{\left[t_{i}\right]_{M}: 1 \leq i \leq M\right\}, \\
& \mathbf{A}=\left\{\left[A_{i}\right]_{M}: 1 \leq i \leq M\right\} .
\end{aligned}
$$




\section{Model of the WCDMA radio interface}

An isolated WCDMA radio interface has a capability of obtaining large theoretical capacities. In real-life conditions, its capacity is, however, constrained by admissible level of interference strength in the frequency channel. Hence, the WCDMA interface is called a noise-limited system or a system with soft capacity [2]. In each mobile system with a dispersion of the signal spectrum, the capacity of the radio interface is limited following an occurrence of a number of types of interference noise, most notably interference from users being serviced in neighboring cells. In traffic considerations, the effect of interference influence of a connection in a neighboring cell upon the cell under consideration (the so-called access cell) is characterised by a decrease in the accessibility of resources for the subscriber of the access cell [2]. Therefore, a call serviced in a neighboring cell "takes away" the resources of both this cell and the access cell. In modelling noise-limited systems, it is possible then to use the approach in which the assumption is that a call of a given class generated in a neighboring cell is serviced simultaneously in a neighboring cell, where it occupies $\left[t_{i}\right]_{M}$ AUs, and in the access cell with a decreased number of AUs equal to $\left[t_{i}\right]_{M}^{\prime}$ AUs. In this case, parameter $\left[t_{i}\right]_{M}^{\prime}$ is the measure of the "noise load," imposed on the access cell by a serviced call generated in a neighboring cell. In descriptions of the UMTS system, the influence of interference noise from other cells is determined by coefficient $\delta$, defined as the ratio between the interference from other cells and the interference of its own [1]. Parameter $\delta$ can be expressed by the following dependence [28]:

$$
\delta=\frac{\sum_{i=1}^{M} \sum_{k=1}^{S}\left[t_{i}\right]_{M}^{\prime} N_{i, k}}{\sum_{i=1}^{M}\left[t_{i}\right]_{M} N_{i}},
$$

where $S$ is the number of adjacent (neighboring) cells to the access cell, $N_{i, k}$ is the average value of the number of serviced calls of class $i$ in the neighboring cell with the index $k(1 \leq k \leq S)$, and $N_{i}$ is the average value of the number of serviced calls of class $i$ in the access cell.

Parameter $\delta$ can then be treated as a fraction that determines the decrease in the availability of the access cell due to the introduction of an "additional" interference load from the serviced subscribers of neighboring cells. Following the adoption of such an interpretation, the availability of the WCDMA interface (uplink and downlink) can be determined by the following formulas [28]:

$$
\begin{aligned}
& d_{\mathrm{UP}}=(1-\delta) V, \\
& d_{\mathrm{DL}}=(1-\delta+\xi) V,
\end{aligned}
$$

where $\xi$ is the interference suppression coefficient, while $\delta$ is the average value of the coefficient adopted by a given operator of a mobile network worked out on the basis of measurements. It indicates the degree of interference reduction between the users of the same cell due to the application of channel codes based on orthogonal variable spreading factor (OVSF). It means that they can have a different dispersion coefficient, and their mutual correlation is zero [41]. The parameter $V$, just as in the previous case, defines the potential theoretical capacity of the isolated radio interface.

To model the occupancy distribution in the WCDMA radio interface, the multi-service model of Erlang's ideal grading (EIG) can be used. Let us consider an EIG to which a mixture of call streams $M$ with different bit rates is offered. The group is defined by the demands of individual call classes $\left[t_{i}\right]_{M}(1 \leq i \leq M)$ expressed in AUs, capacity $V$ expressed in AUs, availability $d$ also expressed in AUs, as well as the traffic intensities $\left[A_{i}\right]_{M}(1 \leq i \leq M)$ of individual call classes, expressed in Erlangs. In the case of the WCDMA interface, parameters $\left[t_{i}\right]_{M}$, and $V$ are defined by Formulas $7-8$, whereas parameter $d$-depending on the type of link-by Formulas 15 or 16. Traffic intensity for calls of particular classes is defined by Formula 4 .

The EIG group is a particular instance of a non-fullavailability groups $[14,29]$ that was defined by Erlang for a single-service case as early as in the beginning of the twentieth century [30]. An approximated model of the group for a multi-service case is proposed in [13]. In [28], this model is used for the first time for traffic analysis of the WCDMA radio interface. The multi-service EIG group is characterized by the following properties:

- The number of inputs to a system $g$ (called load groups) is equal to the number of the possible effective ways of the selection of $d$ AUs from among all $V$ AUs (two load groups differ from each other in at least one $\mathrm{AU}$ ),

- Each load group has access to the same number of $d$ AUs of the group,

- Traffic offered to each of the load groups is identical, and

- The occupancy distribution in each of the load groups is identical.

Figure 2 shows an EIG with the capacity $V=3$ AUs and the availability $d=2$. The group is offered two call classes with the demands respectively equal to $\left[t_{1}\right]_{2}=1$ and $\left[t_{2}\right]_{2}=2$. Observe that the number of load groups is equal to $g=\left(\begin{array}{l}n \\ k\end{array}\right)=3$, in line with the assumptions, while the traffic offered to each of the load groups is identical and equal to $\left[A_{1}\right]_{2} / g$ and $\left[A_{2}\right]_{2} / g$, respectively.

The occupancy distribution in EIG can be determined recursively on the basis of the following formula $[13,14]$ :

$$
n\left[P_{n}\right]_{V}=\sum_{i=1}^{M}\left[A_{i}\right]_{M}\left[t_{i}\right]_{M}\left\{1-\sigma_{i}\left(n-\left[t_{i}\right]_{M}\right)\right\}\left[P_{n-\left[t_{i}\right]_{M}}\right]_{V},
$$




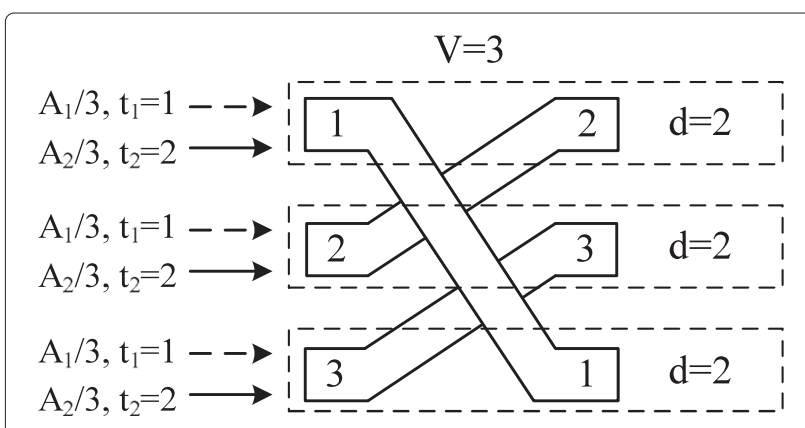

Fig. 2 An exemplary non-full-availability group

where $\left[P_{n}\right]_{V}$ is the occupancy probability of $n$ AUs in the WCDMA interface $(0 \leq n \leq V)$ and $\sigma_{i}(n)$ is the conditional transition probability between the neighboring states of the service process for calls of class $i$ (i.e., states $n$ and $\left.n+\left[t_{i}\right]_{M}\right)$ :

$$
\sigma_{i}(n)=1-\gamma_{i}(n) .
$$

Parameter $\gamma_{i}(n)$ is the conditional blocking probability of calls of class $i$ in the occupancy state of $n$ AUs in EIG. Since-according to the adopted assumptions-the occupancy distributions in load groups are identical, then the conditional blocking probability in one load group of EIG is equal to the conditional blocking probability in the whole group:

$$
\gamma_{i}(n)=\sum_{d-\left[t_{i}\right]_{M}+1}^{\Psi} P_{V, d}(n, x),
$$

where $\Psi=n$ if $\left(d-\left[t_{i}\right]_{M}+1\right) \leq n \leq d$ and $\Psi=d$ if $n>d$.

Probability $P_{V, d}(n, x)$ is the occupancy probability of $x$ AUs in a given load group, defined with the assumption that there are $n$ AUs occupied in the whole EIG. In $[13,14]$, the assumption is that the distribution $P_{[V, d}(n, x)$ is a hypergeometric distribution:

$$
P_{V, d}(n, x)=\left(\begin{array}{l}
d \\
x
\end{array}\right)\left(\begin{array}{l}
V-d \\
n-x
\end{array}\right) /\left(\begin{array}{l}
V \\
n
\end{array}\right) .
$$

Having determined the conditional blocking probabilities $\gamma_{i}(n)$, it is possible to determine the blocking probability for calls of class $i$ in EIG:

$$
\left[E_{i}\right]_{M}=\sum_{n=d-\left[t_{i}\right]_{M}+1}^{V}\left[P_{n}\right]_{V} \gamma_{i}(n) .
$$

In order to simplify the further considerations, the blocking probability for each traffic class, obtained on the basis of the model described by Formulas 17-21, will be presented in the form of the following functional dependence:

$\mathbf{E}_{\mathrm{WCDMA}}=\left\{\left[E_{i, \mathrm{WCDMA}}\right]_{M}: 1 \leq i \leq M\right\}=\operatorname{EIG}(\mathbf{A}, \mathbf{t}, V, d, M)$, where $\mathbf{A}$ and $\mathbf{t}$ are sets of demands and traffic intensities of individual call classes described by Formulas 12 and 13. Let us note that when the availability of the system becomes equal to its capacity $(d=V)$, then the Erlang's ideal grading becomes a full-availability group, described by the ependences (9) and (10). We can thus write:

$$
\operatorname{EIG}(\mathbf{A}, \mathbf{t}, V, V, M)=\operatorname{FAG}(\mathbf{A}, \mathbf{t}, V, M) \text {. }
$$

\section{Model of a system with priorities}

In $[5,6]$, a methodology for modelling of FAG with priorities is developed. In the present section, this methodology will be expanded to include EIG and used to model interfaces in the access part of the UMTS network in which priorities have been introduced.

Let us first consider a system to which two classes of calls are offered, one having a higher priority. This means that the appearance of a new call with a higher priority (class one) may enforce, in the absence of free resources, a termination of the service currently given to calls with a lower priority (class two). As a result of the thus executed method of servicing calls in the system, class two traffic, the one with the lower priority, has no influence on the blocking probability of calls of the first class (i.e., the one with the higher priority). The sets $\mathbf{A}$ and $\mathbf{t}$ for this system are defined as follows:

$$
\begin{aligned}
\mathbf{A} & =\left\{\left[A_{1}\right]_{2},\left[A_{2}\right]_{2}\right\}, \\
\mathbf{t} & =\left\{\left[t_{1}\right]_{2},\left[t_{2}\right]_{2}\right\} .
\end{aligned}
$$

In order to analyze EIG with priorities, let us present the following reasoning that has formerly been used in modelling FAG with priorities [5]. First, we will consider an EIG that services calls with higher priorities only (class 1). Using the already adopted notation-Formulas 24,25 , and 22-we can write:

$$
\left\{\left[E_{1}\right]_{1}\right\}=\operatorname{EIG}\left(\left\{\left[A_{1}\right]_{2}\right\},\left\{\left[t_{1}\right]_{2}\right\}, V, d, 1\right) .
$$

The total traffic $[Y]_{1}$ carried in the system will thus be equal to:

$$
[Y]_{1}=\left[Y_{1}\right]_{1}=\left[A_{1}\right]_{2}\left[t_{1}\right]_{2}\left(1-\left[E_{1}\right]_{1}\right) .
$$

Further, we will consider a case in which EIG services two classes of calls without priorities. The blocking probabilities of individual call classes can be written within the adopted notation in the following way:

$$
\left\{\left[E_{1}\right]_{2},\left[E_{2}\right]_{2}\right\}=\operatorname{EIG}(\mathbf{A}, \mathbf{t}, V, d, 2) .
$$

The total carried traffic in the system becomes:

$$
[Y]_{2}=\sum_{i=1}^{2}\left[Y_{i}\right]_{2}=\sum_{i=1}^{2}\left[A_{i}\right]_{2}\left[t_{i}\right]_{2}\left(1-\left[E_{i}\right]_{2}\right) \text {. }
$$

In Eq. 29, parameter $[Y]_{2}$ defines the total traffic carried in a system that services two classes of calls, whereas $\left[Y_{i}\right]_{2}$ defines the carried traffic of class $i$ in a system that services two classes of calls. 
Now, taking into consideration the results obtained earlier, we can pass on to an analysis of EIG that services two classes of calls with the first class having priority. The blocking probability of the first class $\left[E_{1}\right]_{2}^{P}$ and the carried traffic of the first class $\left[Y_{1}\right]_{2}^{P}$ in the system with priorities will be equal to the blocking probability $\left[E_{1}\right]_{1}$ and carried traffic $\left[Y_{1}\right]_{1}$, respectively, in the system in which only the calls of the first class are serviced. Such equality results from the adopted assumptions according to which the service of calls of the second class with a lower priority does not influence the service of calls of the first class with a higher priority. We can thus write:

$$
\begin{aligned}
& {\left[E_{1}\right]_{2}^{P}=\left[E_{1}\right]_{1},} \\
& {\left[Y_{1}\right]_{2}^{P}=\left[Y_{1}\right]_{1} .}
\end{aligned}
$$

The operation of the system with priorities assumes that in the case of the absence of free resources in the system, calls with higher priorities "enforce" the termination of calls with lower priorities. One can assume then that the traffic conservation principle, according to which the total traffic carried in the system with priorities is the same as in the system without priorities, applies.

$$
\left[Y_{1}\right]_{2}^{P}+\left[Y_{2}\right]_{2}^{P}=[Y]_{2} \text {. }
$$

The relationship between the carried traffic of the second class $\left[Y_{2}\right]_{2}^{P}$ and the offered traffic $\left[A_{2}\right]_{2}$ is determined by the following dependence:

$$
\left[Y_{2}\right]_{2}^{P}=\left[A_{2}\right]_{2}\left[t_{2}\right]_{2}\left(1-\left[E_{2}\right]_{2}^{P}\right) \text {. }
$$

Equations 27 and 30-33 allow us to determine the blocking probability of calls in the system with two priorities, on the basis of the parameters determined in EIG without priorities:

$$
\begin{gathered}
{\left[E_{1}\right]^{P}=\left[E_{1}\right]_{1}} \\
{\left[E_{2}\right]^{P}=\left[E_{2}\right]_{2}^{P}=\frac{\left[A_{1}\right]_{2}\left[t_{1}\right]_{2}\left(\left[E_{1}\right]_{2}-\left[E_{1}\right]_{1}\right)+\left[A_{2}\right]_{2}\left[t_{2}\right]_{2}\left[E_{2}\right]_{2}}{\left[A_{2}\right]_{2}\left[t_{2}\right]_{2}} .}
\end{gathered}
$$

Let us now consider a generalized case in which each class of call has an appropriate priority assigned. Let us assume that the system services $M$ call classes. The serviced call classes have been arranged (indexed) from the highest priority to the lowest priority (the class with index $i$ has a lower priority than the class with index $i-1)$. The determination of the blocking probability in EIG with $M$ priorities can be reduced to an $M-1$ step computational algorithm in which in every step of the algorithm a system with two priorities is taken into consideration. In the first step, class $M$ (according to the indexing system, has a lower priority, whereas the remaining $M-1$ classes form classes with a higher priority and "push out" traffic of class
$M$. In the successive steps, traffic of class $i$ has the lowest priority, whereas traffic of the remaining $i-1$ classes forms the class with the higher priority. In the last step, we consider the traffic of the call classes $\left[A_{1}\right]_{M}$ and $\left[A_{2}\right]_{M}$ by determining appropriate characteristics of the considered traffic using directly Formulas 34 and 35. By applying exactly the same reasoning as in the case of the two priorities, we can determine the blocking probability for calls of class $i(1 \leq i \leq M)$ in EIG with $M$ priorities:

$$
\left[E_{i}\right]_{M}^{P}=\left[E_{i}\right]_{i}^{P}=\frac{\sum_{r=1}^{i-1}\left[A_{r}\right]_{i}\left[t_{r}\right]_{i}\left(\left[E_{r}\right]_{i}-\left[E_{r}\right]_{i-1}\right)+\left[A_{i}\right]_{i}\left[t_{i}\right]_{i}\left[E_{i}\right]_{i}}{\left[A_{i}\right]_{i}\left[t_{i}\right]_{i}} .
$$

The exemplary algorithm that makes it possible to determine all blocking probabilities in EIG with priorities can be written in the form of algorithm ALG_EIG_P(A,t $, V, d, \mathbf{M})$, presented below.

\section{Algorithm 1 The algorithm ALG_EIG_P $(\mathbf{A}, \mathbf{t}, V, d, M)$. \\ 1. Setting the initial value $i=M$, according to the hierarchy of the traffic classes: $1,2, \ldots, M$, \\ 2. Determination of the blocking probability for call classes from 1 to $i-1$ using the EIG Models 17-22:

$$
\left\{\left[E_{r}\right]_{i-1}: 1 \leq r \leq i-1\right\}=\operatorname{EIG}(\mathbf{A}, \mathbf{t}, V, d, i-1) .
$$

3. Determination of the blocking probability for call classes from 1 to $i$ using the EIG Models 17-22:

$$
\left\{\left[E_{r}\right]_{i}: 1 \leq r \leq i-1\right\}=\operatorname{EIG}(\mathbf{A}, \mathbf{t}, V, d, i) .
$$

4. Determination of the blocking probability for calls of class $i$ in EIG with priorities on the basis of Formula 36:

$$
\left[E_{i}\right]_{M}^{P}=\left[E_{i}\right]_{i}^{P}=\frac{\sum_{r=1}^{i-1}\left[A_{r}\right]_{i}\left[t_{r}\right]_{i}\left(\left[E_{r}\right]_{i}-\left[E_{r}\right]_{r-1}\right)+\left[A_{i}\right]_{i}\left[t_{i}\right]_{i}\left[E_{i}\right]_{i}}{\left[A_{i}\right]_{i}\left[t_{i}\right]_{i}} .
$$

5. Decrease in the value of the variable $i: i=i-1$,

6. Checking of the variable value $i$ :

$i \geq 2$; proceed to step 2 ,

$i<2 ;\left[E_{1}\right]_{M}^{P}=\left[E_{1}\right]_{1}$; termination of calculations.

\section{Model of setting up connections in the access part of UMTS}

Let us consider the operation of the telecommunication system presented in Fig. 3 that is composed of two smaller sub-systems (the so-called component systems). Each component system has its own resources to which a common (shared) call stream is offered. A new call can be admitted for service only when this call can be admitted for service in both component systems. This means that the Sys2 system is offered only to those calls that have been rejected by Sys1. In the same way, Sys1 is offered to calls that have not been rejected in Sys2. To analyze such 


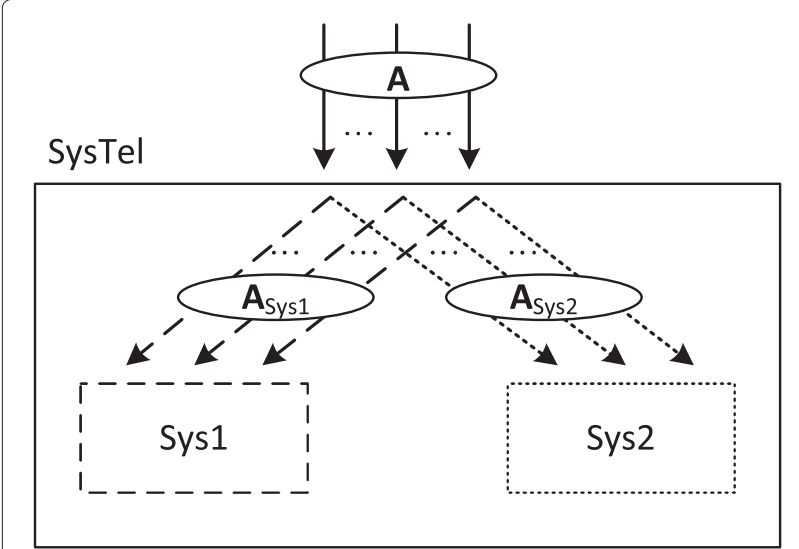

Fig. 3 The idea of the fixed-point methodology

complex systems, the fixed-point methodology is used [42].

Our further assumption is that the component systems employ independently the priority mechanism described in Section 7. This means that a given class of calls may have a different priority in each of the component systems and, in consequence, a different record number (index). Moreover, the organization of classes in both component systems may differ from the one that was originally adopted. To normalize the notation of the proposed method, the following representation has been adopted:

$$
\text { SysTel }=\{\text { A, c, } M, \text { Sys1, Sys2 }\},
$$

where:

- A-Traffic offered to the system under consideration:

$$
\mathbf{A}=\left\{\left[A_{i}\right]_{M}: i \in\{1,2, \ldots, M\},\right.
$$

- $\mathbf{c}-$ Resources demanded by calls of individual classes:

$$
\mathbf{c}=\left\{\left[c_{i}\right]_{M}: i \in\{1,2, \ldots, M\}\right\},
$$

- Sys $X-C o m p o n e n t$ system $X(X \in\{1,2\})$ :

$$
\text { Sys } \mathbf{X}=\left\{\mathbf{A}_{\text {SysX }}, \mathbf{t}_{\text {SysX }}, V_{\text {SysX }}, M\right\},
$$

where:

- $\mathbf{A}_{\text {SysX }}$ - Traffic offered to system SysX (the sequence of classes is enforced by the priority mechanism in the system):

$$
\mathbf{A}_{\text {SysX }}=\left\{\left[A_{j, \text { SysX }}^{i}\right]_{M}: j \in\{1,2, \ldots, M\}\right\},
$$

- $\left[A_{j, \text { SysX }}^{i}\right]_{M}-$ Traffic of class $i$ with priority $j$ in system SysX,

- $\mathbf{t}_{\text {Sys }} \mathrm{X}$-Resources demanded by calls of individual classes expressed in allocation units determined in relation to the type of system SysX:

$$
\mathbf{t}_{\text {SysX }}=\left\{\left[t_{j, \text { Sys }}^{i}\right]_{M}: j \in\{1,2, \ldots, M\}\right\},
$$

- $\left[t_{j, \text { Sys X }}^{i}\right]_{M}-$ Resources demanded by calls of class $i$ with priority $j$ in system SysX, expressed in units for system SysX, and

- $V_{\text {SysX }}-$ The capacity of system SysX, expressed in units for this system.

The effect of the correlation of service processes in both systems in the fixed-point methodology is taken into account by the introduction of the notion of effective traffic. According to the definition [42], effective traffic offered to the first system is exactly the traffic that is not lost in the second system. Therefore, the intensity $\left[A_{j, \text { Sys } 1}^{i}\right]_{M}$ of the effective traffic of class $i$, offered in system Sys1 with priority $j$, and the intensity $\left[A_{k, \text { Sys } 2}^{i}\right]_{M}$ of the effective traffic of class $i$, offered in system Sys 2 with priority $k$, can be written in the following way:

$$
\begin{aligned}
& {\left[A_{j, \text { Sys } 1}^{i}\right]_{M}=\left[A_{i}\right]_{M}\left(1-\left[E_{k, \text { Sys } 2}^{i}\right]_{M}\right),} \\
& {\left[A_{k, \text { Sys } 2}^{i}\right]_{M}=\left[A_{i}\right]_{M}\left(1-\left[E_{j, \text { Sys } 1}^{i}\right]_{M}\right),}
\end{aligned}
$$

where $\left[E_{j, \text { Sys } 1}^{i}\right]_{M}$ and $\left[E_{k, \text { Sys } 2}^{i}\right]_{M}$ is the blocking probability for calls of the same class $i$, indexed differently in Sys1 $(1 \leq$ $j \leq M)$ and in Sys2 $(1 \leq k \leq M)$.

In line with the hierarchy of priorities for individual call classes conventionally adopted for these systems, the total blocking probability for calls of class $i$ in the tandem Sys1Sys2 in the fixed-point methodology can be expressed by the following formula:

$$
\left[E_{i}\right]_{M}=1-\left(1-\left[E_{j, \text { Sys } 1}^{i}\right]_{M}\right)\left(1-\left[E_{k, \text { Sys } 2}^{i}\right]_{M}\right) .
$$

The calculations of the probabilities in Eq. 45 are performed iteratively until the required level of a relative error has been reached. In each consecutive step, new intensities of effective traffic and the corresponding blocking probabilities are determined. The method for the determination of the blocking probability depends on the adopted model of the system, e.g., there is a choice of the FAG model for the Iub interface (Formulas 9-10) or the EIG model for the WCDMA interface (Formulas 17-22). In the case of the application of priorities in determining the blocking probability of individual call classes in composed (complex) systems (e.g., WCDMA and Iub interfaces), it is possible to use the model proposed in Section 7 and the algorithm ALG_EIG_P(A,t,d,V,M) worked out on its basis. 
Given below is a simplified algorithm (ALG_FPM(Sys1, Sys2)) for the calculation of the blocking probability in the tandem of the two systems Sys1 and Sys2.

Algorithm 2 The algorithm ALG_FPM (Sys1, Sys2).

1. Parameterization of systems

$$
\begin{aligned}
& \text { SysTel }=\{\mathbf{A}, \mathbf{c}, M, \text { Sys1, Sys2 }\}, \\
& \text { Sys1 }=\left\{\mathbf{A}_{\mathrm{Sys} 1}, \mathbf{t}_{\mathrm{Sys} 1}, V_{\mathrm{Sys} 1}, M\right\}, \\
& \text { Sys2 }=\left\{\mathbf{A}_{\mathrm{Sys} 2}, \mathbf{t}_{\mathrm{Sys} 2}, V_{\mathrm{Sys} 2}, M\right\} .
\end{aligned}
$$

2. Setting the initial value for the iteration step $k=0$ and the assumption that:

$$
\begin{aligned}
& \left\{\left[E_{j, \text { Sys } 1}^{i}\right]_{M}^{(0)}, 1 \leq j \leq M\right\}=0, \\
& \left\{\left[E_{k, \text { Sys } 2}^{i}\right]_{M}^{(0)}, 1 \leq k \leq M\right\}=0 .
\end{aligned}
$$

3. Determination of effective traffic for calls of all classes in the first and the second system:

$$
\begin{gathered}
{\left[A_{j, \text { Sys } 1}^{i}\right]_{M}^{(l)}=\left[A_{i}\right]_{M}\left(1-\left[E_{k, \text { Sys } 2}^{i}\right]_{M}^{(l-1)}\right),} \\
{\left[A_{k, \text { Sys } 2]_{M}^{i}}^{(l)}=\left[A_{i}\right]_{M}\left(1-\left[E_{j, \text { Sys } 1}^{i}\right]_{M}^{(l-1)}\right) .\right.}
\end{gathered}
$$

4. Determination of the blocking probability for calls of all classes in the first and the second system:

$$
\begin{aligned}
& \left\{\left[E_{j, \text { Sys } 1}^{i}\right]_{M}^{(l)}, 1 \leq j \leq M\right\}= \\
& \text { ALG_EIG_P(A } \left.\mathbf{A}_{\text {Sys } 1}, \mathbf{t}_{\text {Sys } 1}, V_{\text {Sys } 1}, \mathrm{~d}_{\text {Sys } 1}, M\right) \text {, } \\
& \left\{\left[E_{k, \text { Sys2 } 2}^{i}\right]_{M}^{(l)}, 1 \leq k \leq M\right\}= \\
& \text { ALG_EIG_P(A } \left.\mathbf{A}_{\text {Sys2 } 2}, \mathbf{t}_{\text {Sys } 2}, V_{\text {Sys } 2}, \mathrm{~d}_{\text {Sys2 }}, M\right) \text {. }
\end{aligned}
$$

5. Determination of the blocking probability for calls of all classes in the tandem of the two systems:

$$
\left[E_{i}\right]_{M}^{(l)}=1-\left(1-\left[E_{j, \text { Sys } 1}^{i}\right]_{M}^{(l)}\right)\left(1-\left[E_{k, \text { Sys } 2}^{i}\right]_{M}^{(l)}\right) .
$$

6. Checking the accuracy of calculations for each class of calls $i(1 \leq i \leq M)$ :

$$
\left|\frac{\left[E_{i}\right]_{M}^{(l-1)}-\left[E_{i}\right]_{M}^{(l)}}{\left[E_{i}\right]_{M}^{(l)}}\right| \leq \varepsilon,
$$

the condition is not satisfied; $l=l+1$, proceed to step 2, the condition is satisfied; $\left[E_{i}\right]_{M}=\left[E_{i}\right]_{M}^{(l)}$, termination of calculations.

In the algorithm presented above, the following notation has been adopted: symbol $\left[X_{j, \text { Sys } 1}^{i}\right]_{M}^{(l)}$ defines the value of the $X$ parameter in the $l$ th step of the iteration.
Let us now consider the sequence of calculations in a telecommunication system composed of the WCDMA interface and the Iub interface in tandem, assuming that in each interface, a mechanism has been introduced for the prioritization of $M$ classes of calls. Another assumption is that the hierarchy of priorities in each of the interfaces can be different. This means that call classes offered to both interfaces are appropriately arranged (WCDMA interface $1 \leq j \leq M$, Iub interface $1 \leq k \leq M)$. The calculations start with the determination of AU in each of the interfaces. Then, the sets of demands of the number of AUs by calls of particular classes $\mathbf{t}_{\mathrm{WCDMA}}$ and $\mathbf{t}_{\text {Iub }}$ in respective interfaces are determined. The capacities and availabilities of the considered interfaces are also expressed in AUs. After the parameterization of the interfaces, on the basis of the fixed-point methodology and the algorithm ALG_EIG_P $(\mathbf{A}, \mathbf{t}, \mathrm{V}, \mathrm{d}, \mathrm{M})$ for each interface, it is possible to determine the blocking probability for calls of individual classes in the tandem of WCDMA and Iub. Given below is a simplified algorithm for the calculations of the blocking probability in the tandem.

\section{Case study}

The results of the analytical studies have been confirmed by the results of the simulation that was carried out based on an original simulation program developed by the authors. The simulator was implemented in the Python language, and it employed the event scheduling approach [43]. The simulation model, however, does not take into consideration all technological parameters for the UMTS system. For example, it takes into account neither the propagation model of the radio channel nor the mobility of users. The developed simulator is intended to model the traffic capacity of the system, and thus to model the system at the so-called call (flow or connection) level [38]. At this level, technological parameters are rather insignificant for the accuracy of mapping the system to be modelled [44]. In the simulation experiments presented in all included graphs, each point on the curve represents an average value of the blocking probability obtained in the fifth series of the simulation. The assumption was that in an individual simulation series, at least 10,000,000 calls of the class that required the highest number of allocation units for service were offered. The results of the simulation are presented in graphs in the form of marks with a $95 \%$ confidence level determined and $5 \%$ confidence interval on the basis of the Student's $t$-distribution. In many instances, the values of the confidence intervals are almost included inside the marks that identify the average result of the simulation experiment for calls of a given traffic class.

In order to validate the proposed analytical models, the possibility of an applying priority mechanisms for a differentiation of the service level for subscribers of the 
Algorithm 3 Algorithm for the determination of the blocking probability in the tandem WCDMA interface-Iub interface.

1. Determination of the sets of parameters describing the call stream offered to the considered section of the UMTS network (Eqs. 38 and 39).

2. Appropriation of real systems from the considered section of the UTMS network to systems Sys1 and Sys2: Sys1 = WCDMA interface; Sys2 = Iub interface;

3. Determination of the sets of parameters that describe the WCDMA interface:

(a) Expression of demands of individual call classes in noise units:

$$
L_{\mathrm{WCDMA}}=\left\{\left[L_{1}\right]_{M}, \ldots,\left[L_{i}\right]_{M}, \ldots,\left[L_{M}\right]_{M}\right\} .
$$

(b) Setting the value of AU for the WCDMA interface (Formula 6)

$$
L_{\mathrm{AU}}=\mathrm{GCD}\left(\left[L_{1}\right]_{M}, \ldots,\left[L_{i}\right]_{M}, \ldots,\left[L_{M}\right]_{M}\right) .
$$

(c) Determination of sets of demands of the number of AUs by calls of individual classes (Formula 7):

$$
\mathbf{t}_{\mathrm{WCDMA}}=\left\{t_{i, \mathrm{WCDMA}}=\left\lceil L_{i} / L_{\mathrm{AU}}\right\rceil: 1 \leq i \leq M\right\} .
$$

(d) Determination of the capacity of the WCDMA interface (Formula 8), expressed in AUs:

$$
V_{\mathrm{WCDMA}}=\left\lfloor 1 / L_{\mathrm{AU}}\right\rfloor \text {. }
$$

(e) Determination of indexes of call classes resulting from the priority mechanism adopted in the WCDMA interface (Eqs. 41 and 42):

$$
\begin{aligned}
& \mathbf{A}_{W C D M A}=\left\{\left[A_{j, \mathrm{WCDMA}}^{i}\right]_{M}: j \in\{1,2, \ldots, M\}\right\}, \\
& \mathbf{t}_{\mathrm{WCDMA}}=\left\{\left[t_{j, \mathrm{WCDMA}}^{i}\right]_{M}: j \in\{1,2, \ldots, M\}\right\} .
\end{aligned}
$$

(f) Determination of the availability of the WCDMA interface (Formula 15):

$$
d_{\mathrm{WCDMA}}=(1-\delta) V_{\mathrm{WCDMA}} \text {. }
$$

4. Determination of sets of parameters describing the system Iub interface:

(a) Setting the value of AU for the Iub interface (Formula 1)

$$
c_{\mathrm{AU}}=\mathrm{GCD}\left(\left[c_{1}\right]_{M}, \ldots,\left[c_{i}\right]_{M}, \ldots,\left[c_{M}\right]_{M}\right) .
$$

(b) Determination of sets of demands of the number of AUs by calls of individual classes (Formula 2):

$$
\mathbf{t}_{\text {Iub }}=\left\{t_{i, \text { Iub }}=\left\lceil c_{i} / c_{\mathrm{AU}}\right\rceil: 1 \leq i \leq M\right\} .
$$

(c) Determination of capacities of the Iub interface (Formula 3), expressed in AUs:

$$
V_{\text {Iub }}=\left\lfloor C / c_{\mathrm{AU}}\right\rfloor \text {. }
$$

(d) Determination of indexes of call classes resulting from the priority mechanism adopted in the Iub interface (Eqs. 41 and 42):

$$
\begin{aligned}
& \mathbf{A}_{\text {Iub }}=\left\{\left[A_{k, \text { Iub }}^{i}\right]_{M}: k \in\{1,2, \ldots, M\}\right\}, \\
& \mathbf{t}_{\text {Iub }}=\left\{\left[t_{k, \text { Iub }}^{i}\right]_{M}: k \in\{1,2, \ldots, M\}\right\} .
\end{aligned}
$$

(e) Determination of the availability of the Iub interface (Formula 15):

$$
d_{\text {Iub }}=V_{\text {Iub }} \text {. }
$$

5. Determination of the blocking probability for individual call classes in the tandem WCDMA interface-Iub interface on the basis of the fixed-point methodology:

$$
\mathbf{E}=\text { ALG_FPM(WCDMA, Iub). }
$$


UMTS network was considered. In the case of the UMTS network, traffic management functions in radio network controller (RNC) is used for this purpose. The differentiation may influence the service parameters of the traffic flow through the Iub interface and the WCDMA radio interface. The following assumptions were then adopted in the study:

- A priority is assigned independently to calls of each service classes,

- In the case of the lack of free resources, the arrival of a call with a higher priority can be followed by the termination of service for calls with a lower priority, and

- Setting up a connection requires the simultaneous provision of free resources in the radio interface and in the Iub interface.

Analytical calculations were performed according to Algorithm 3, while the accuracy of the model was validated by the comparison of corresponding results with the results of simulation experiments.

The assumption was that the considered system was composed of six cells that were connected to the backbone network via the Iub interface with a bit rate of $15 \mathrm{Mbps}$ (Fig. 4). It was adopted that each cell serviced four classes of service: class 1 -emergency calls, class 2-voice, class 3 -data 1 (64 kbps), and class 4-data 2 (384 kbps). Calls of class $i$ demanded the following number of allocation units [45]:

$$
\begin{aligned}
& t_{\mathrm{Iub}, 1}=16 \mathrm{AU}, t_{\mathrm{WCDMA}, 1}=53 \mathrm{AU} \\
& t_{\mathrm{Iub}, 2}=16 \mathrm{AU}, t_{\mathrm{WCDMA}, 2}=53 \mathrm{AU} \\
& t_{\mathrm{Iub}, 3}=64 \mathrm{AU}, t_{\mathrm{WCDMA}, 3}=257 \mathrm{AU} \\
& t_{\mathrm{Iub}, 4}=384 \mathrm{AU} t_{\mathrm{WCDMA}, 4}=503 \mathrm{AU} .
\end{aligned}
$$

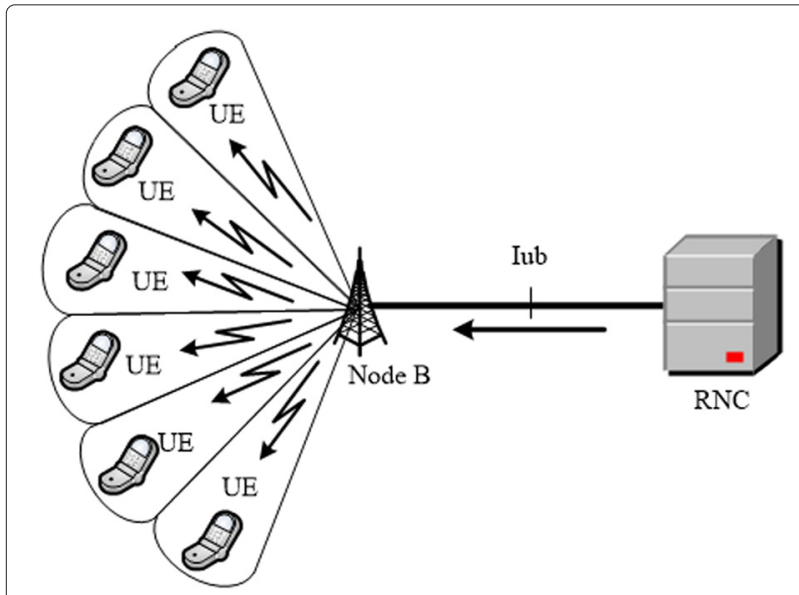

Fig. 4 Scheme of the exemplary system

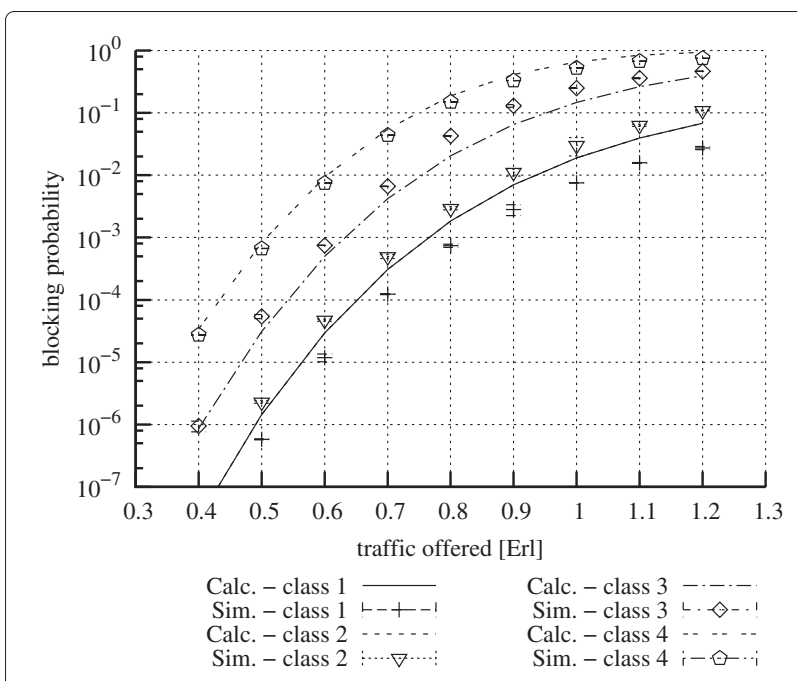

Fig. 5 Blocking probability in WCDMA-lub system without priorities (class 1 -emergency calls, class 2-voice, class 3-data (64 kbps), and class 4-data (384 kbps))

Another assumption was that traffic of individual classes was offered in the following proportions:

$$
A_{1} t_{1}: A_{2} t_{2}: A_{3} t_{3}: A_{4} t_{4}=1: 15: 4: 2 .
$$

The hierarchy of priorities was adopted to be identical in both interfaces under consideration as well as consistent with the indexing of call classes given above. The capacity of the radio interface in the uplink direction was $8000 \mathrm{AU}$, whereas the capacity of the Iub interface was 15,000 AU.

Figures 5, 6, and 7 show the dependence between blocking probability and traffic intensity offered per one allocation unit in the WCDMA interface. Figure 5 presents the

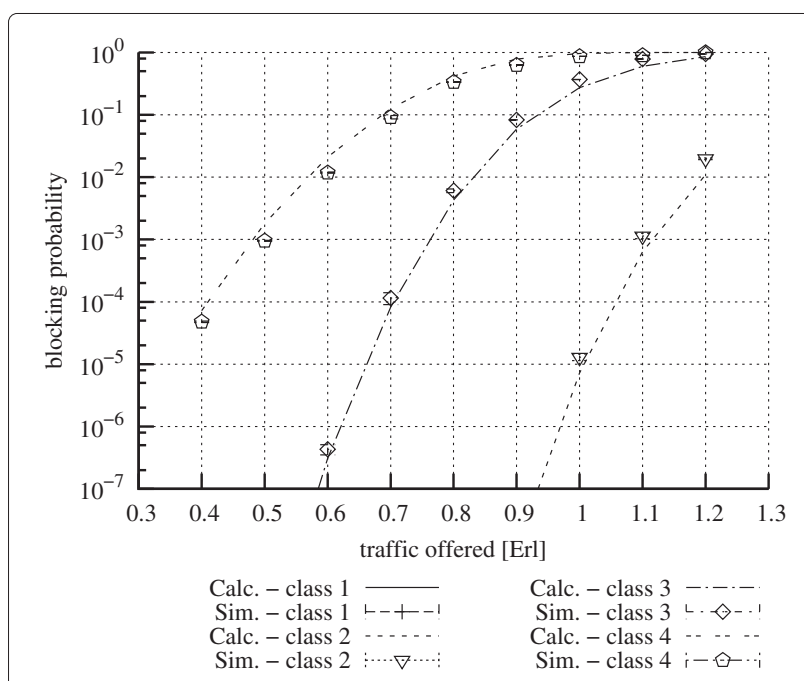

Fig. 6 Blocking probability in WCDMA-lub system with priorities (class 1 -emergency calls, class 2-voice, class 3-data (64 kbps), and class 4-data (384 kbps)) 


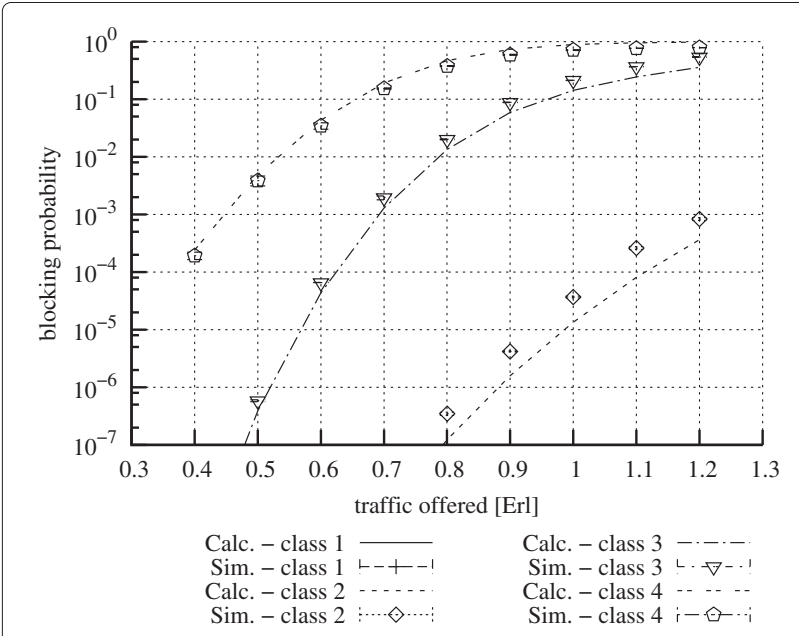

Fig. 7 Blocking probability in WCDMA-lub system with priorities (class 1 -emergency calls, class 2 (3) —data (64 kbps), class 3 (2) — voice, and class 4-data (384 kbps))

results obtained for a system without priorities, whereas Figs. 6 and 7 show the blocking probability in a system with priorities. While comparing the presented graphs, it is easy to notice that the introduction of priorities (Figs. 5, 6, and 7) influences the values of the blocking probability. The blocking probability for classes with higher priority decreases, while the blocking probability for classes with lower priorities increases. This phenomenon is in line with the operational principle of priority traffic management. The arrival process of calls with higher priority can be followed-in the case of the lack of free resources required for the service of a given call-by calls with lower priorities being pushed out.

It can thus be assumed that after the introduction of priorities, the amount of resources available for calls of classes with higher priorities increases and, this way, the amount of resources for calls with lower priorities decreases. An increase or a decrease in the amount of available resources leads to an increase or a decrease in the value of the blocking probability for calls of appropriate traffic classes.

Figure 7 presents the results obtained for a system in which priorities were changed for classes 2 and 3 . It is easily noticeable that despite a higher number of demanded allocation units, class 3 (data) is characterised by a lower blocking probability than class 2 (voice).

While introducing priorities, the operator should take into consideration the fact that an increase in the volume of traffic of the class with the highest priority will result in a decrease in the amount of resources available for calls of the remaining traffic classes. The accuracy of the determination of the values of the blocking probability for particular traffic classes obtained on the basis of the model of a system with priorities is slightly lower than that of the results obtained on the basis of the model of a system without priorities. The difference results from the assumptions adopted with regard to a model in which traffic serviced in a system with priorities is approximated by traffic serviced in a system without priorities. The differences between the values of the blocking probability are generally only slight and it can be stated that the results presented in Figs. 5, 6, and 7 confirm the validity of the assumptions adopted for theoretical models. Additionally, in Table 1, a detailed presentation of the differences between the simulation results and the results of the calculations is given (with relative error $(\delta)$ for results presented in Fig. 6).

The models and algorithms discussed in the article use the most effective approach to modeling multi-service systems that is based on the approximation of the service process that occurs in the system by an appropriate Markov chain. The occupancy distribution in a single multi-service system can be determined on the basis of appropriate recurrent formulas, e.g., $[2,11,12,14]$ that take the specificity of systems into consideration. Given the concurrent nature of the call service in two multiservice systems (radio interface and Iub interface), the article employs the fixed point methodology [42]. This method has already been used in earlier works of the authors (e.g., in [44, 46-50]) in order to take into account the concurrency of service processes that occur in different multi-service systems. The fixed point methodology is characterized by a high effectiveness, e.g., [42]. The decision to drop the application of this method would necessitate the consideration of a very complex Markov process that would correspond to the service process occurring concurrently in both systems. Such an approach would necessitate the use of far more complex mathematical

Table 1 Accuracy of the model (Fig 6)

\begin{tabular}{|c|c|c|c|c|c|c|c|c|c|}
\hline \multirow[t]{2}{*}{$a$} & \multicolumn{3}{|c|}{ Class 2} & \multicolumn{3}{|c|}{ Class 3} & \multicolumn{3}{|c|}{ Class 4} \\
\hline & $E_{2}(\operatorname{sim})$. & $E_{2}$ (calc.) & $\delta[\%]$ & $E_{3}(\operatorname{sim})$. & $E_{3}$ (calc.) & $\delta[\%]$ & $E_{4}(\operatorname{sim})$. & $E_{4}$ (calc.) & $\delta[\%]$ \\
\hline 0.5 & 0 & 0 & 0 & 0 & 0 & 0 & $1.41 E-03$ & $9.52 \mathrm{E}-04$ & 32.7 \\
\hline 0.7 & 0 & 0 & 0 & $8.74 \mathrm{E}-05$ & $1.15 E-04$ & 32 & $1.27 E-0$ & $9.75 \mathrm{E}-02$ & 23.1 \\
\hline 0.9 & 0 & 0 & 0 & $5.90 \mathrm{E}-02$ & 7.27E-02 & 23.1 & $7.86 \mathrm{E}-01$ & $6.91 \mathrm{E}-01$ & 12.2 \\
\hline 1.1 & $6.34 \mathrm{E}-04$ & $4.92 \mathrm{E}-04$ & 22.5 & $6.02 \mathrm{E}-01$ & $7.13 \mathrm{E}-01$ & 18.4 & $9.97 \mathrm{E}-01$ & 8.97E-01 & 10 \\
\hline
\end{tabular}


models that, in practice, would impose limits on the analysis of systems reducing it to the analysis of systems with a very low capacity, much lower than the capacity of the systems considered in the article.

\section{Conclusions}

This paper presents a new analytical model of the part of the UMTS network in which a mechanism for services prioritization has been introduced. The proposed model involves a radio interface and an Iub interface. The paper proposes a new model of the non-full-availability group with priorities that has been used to model the WCDMA interface. To model the Iub interface, in turn, a model of the full-availability group with priorities that has been developed earlier is used. The mutual dependence between the serviced traffic in both interfaces is taken into account by the fixed-point methodology. The model is then used to evaluate the blocking probability in an exemplary system. The results obtained in the study confirm a satisfactory accuracy of the model. This model can be used to analyze dimension and optimize multi-service mobile phone networks.

\section{Endnote}

${ }^{\mathrm{a}}$ In the functional notation the two-index notation $\left[X_{i}\right]_{r}$ has been adopted in which the internal index $i$ defines the current index of a given parameter, while the external index, $r$, indicates the maximum value of index $i$, e.g., as in the notation $\left[c_{i}\right]_{M}$, where $(1 \leq i \leq M)$, the internal index defines the traffic class, whereas the external index $M$ indicates the class with the highest index, which is equivalent to the number of all classes serviced in the system. Thus, the adopted notation will make further considerations more convenient.

\section{Competing interests}

The authors declare that they have no competing interests.

\section{Acknowledgments}

The presented work has been funded by the Polish Ministry of Science and Higher Education within the status activity task "Structure, analysis and design of modern switching system and communication networks" in 2015.

Received: 13 January 2015 Accepted: 1 July 2015

Published online: 18 July 2015

\section{References}

1. H Holma, A Toskala, HSDPAVHSUPA for UMTS: High Speed Radio Access for Mobile Communications. (John Wiley \& Sons, Ltd., Chichester, West Sussex, 2006)

2. M Stasiak, M Głąbowski, A Wiśniewski, P Zwierzykowski, Modeling and Dimensioning of Mobile Networks. (John Wiley \& Sons, Ltd., Chichester, West Sussex, 2010)

3. P Garcia, A Gonzalez, A Alonso, B Martinez, JM Perez, A Esguevillas, Automatic UMTS system resource dimensioning based on service traffic analysis. EURASIP J. Wireless Commun. Netw. 2012(1), 323 (2012)

4. PA Garcia, AA Gonzalez, AA Alonso, B Carro Martinez, JMA Perez, AS Esguevillas, in 21st International Conference on Computer Communications and Networks. A method for the detection of QoS degradation in UMTS networks (Munich, 2012), pp. 1-5
5. M Stasiak, P Zwierzykowski, J Wiewióra, D Parniewicz, in Computer Performance Engineering. Lecture Notes in Computer Science, ed. by N Thomas, C Juziz. An approximate model of the WCDMA interface servicing a mixture of multi-rate traffic streams with priorities, vol. 5261 (Springer, Palma de Mallorca, Spain, 2008), pp. 168-180

6. M Stasiak, J Wiewióra, P Zwierzykowski, in International Symposium on Information Theory and Its Applications, ISITA2008. The analytical model of the WCDMA interface with priorities in the UMTS network (Auckland, New Zealand, 2008)

7. K Subramaniam, AA Nilsson, in IEEE International Conference on Communications (ICC). An analytical model for adaptive call admission control scheme in a heterogeneous UMTS-WCDMA system, vol. 5, (2005), pp. 3334-33385. doi:10.1109/ICC.2005.1495039

8. K Subramaniam, AA Nilsson, in IEEE 61st Vehicular Technology Conference. Tier-based analytical model for adaptive call admission control scheme in a UMTS-WCDMA system, vol. 4, (2005), pp. 2181-21854. doi:10.1109/VETECS.2005.1543721

9. M Stasiak, P Zwierzykowski, J Wiewióra, in Fifth Advanced International Conference on Telecommunications. Analytical model of the WCDMA radio interface in UMTS network with user differentiation (Venice, 2009), pp. 83-88

10. M Stasiak, P Zwierzykowski, J Wiewióra, in International Conference on Telecommunications. WCDMA interface in UMTS network carrying a mixture of multi-rate traffic with different priorities (Marrakech, 2009), pp. 254-259

11. JS Kaufman, Blocking in a shared resource environment. Commun. IEEE Trans. 29(10), 1474-1481 (1981)

12. JW Roberts, in Proceedings of Performance of Data Communications Systems and Their Applications, ed. by G Pujolle. A service system with heterogeneous user requirements-application to multi-service telecommunications systems (North Holland Amsterdam, 1981), pp. 423-431

13. M Stasiak, An approximate model of a switching network carrying mixture of different multichannel traffic streams. IEEE Trans. Commun. 41(6), 836-840 (1993)

14. M Głąbowski, S Hanczewski, M Stasiak, Modeling Erlang's ideal grading with multirate BPP traffic. Mathematical Problems in Engineering (2012). Article ID 456910

15. M Głąbowski, A Kaliszan, M Stasiak, Modeling product-form state-dependent systems with BPP traffic. J. Perform. Eval. 67(3), 174-197 (2010)

16. M Stasiak, Combinatorial considerations for switching systems carrying multi-channel traffic streams. Annales des Télécommunications. 51(11-12), 611-625 (1996)

17. S Hanczewski, M Stasiak, in Proceedings of First International Workshop (SAPIR 2004, Lecture Notes in Computer Science, ed. by P Dini, P Lorenz, and JN Souza. Point-to-group blocking in 3-stage switching networks with multicast traffic streams (Springer, Fortaleza, Brasil, 2004), pp. 219-230

18. M Głąbowski, in Proceedings of Asia-Pacific Conference on Communications. Recurrent calculation of blocking probability in multiservice switching networks (Busan, Korea, 2006), pp. 1-5

19. M Głąbowski, M Stasiak, in Proceedings of 19th International Teletraffic Congress. Analytical model of switching networks with bandwidth reservation and several attempts of setting up a connection (Beijing, China, 2005), pp. 1405-1414

20. M Głąbowski, M Stasiak, Point-to-point blocking probability in switching networks with reservation. Annales des Télécommunications. 57(7-8), 798-831 (2002)

21. M Głąbowski, K Kubasik, M Stasiak, Modeling of systems with overflow multi-rate traffic. Telecommun. Syst. 37(1-3), 85-96 (2008)

22. M Głąbowski, S Hanczewski, M Stasiak, in AFRICON, 2011. Erlang's ideal grading in DiffServ modelling, (2011), pp. 1-6. doi:10.1109/AFRCON.2011.6072139

23. M Głąbowski, MD Stasiak, Modelling of multiservice switching networks with overflow links for any traffic class. Circuits, Devices Syst. 8(5), 358-366 (2014)

24. S Hanczewski, M Stasiak, in 17th Asia-Pacific Conference on Communications. Performance modelling of video-on-demand systems, (2011), pp. 784-788. doi:10.1109/76.931111 
25. S Hanczewski, M Stasiak, in Computer Networks. Communications in Computer and Information Science, ed. by P Gaj, P Stera. Modeling of video on demand systems (Springer, Brunów, Poland, 2014), pp. 233-242

26. M Stasiak, S Hanczewski, in International Symposium on Information Theory and Its Applications. A model of WCDMA radio interface with reservation (Auckland, 2008), pp. 1-6

27. M Stasiak, Wiewió,ra, P Zwierzykowski, D Parniewicz, in Computer Performance Engineering, 6th European Performance Engineering Workshop, EPEW 2009, London, UK, July 9-10, 2009, Proceedings. Analytical model of traffic compression in the UMTS network (London, UK, 2009), pp. 79-93

28. M Stasiak, M Głąbowski, S Hanczewski, in 8th International Symposium on Communication Systems, Networks \& Digital Signal Processing, CSNDSP 2012, Poznan, Poland, July 18-20, 2012. The application of the Erlang's ideal grading for modelling of UMTS cells (Poznan, 2012), pp. 1-6

29. A Lotze, in Proceedings of 5th International Teletraffic Congress. History and development of grading theory (New York, USA, 1967), pp. 148-161

30. E Brockmeyer, A survey of A.K. Erlang's mathematical works. Trans. Dan. Acad. Tech. Sci. 2, 101-126 (1948)

31. M Stasiak, S Hanczewski, in Computer Performance Engineering. Lecture Notes in Computer Science. Approximation for multi-service systems with reservation by systems with limited-availability (Springer, Palma de Mallorca, Spain, 2008), pp. 257-267

32. M Stasiak, S Hanczewski, A new analytical model of UMTS cell. Image Process. Commun. 17(1-2), 81-89 (2013)

33. D Parniewicz, M Stasiak, P Zwierzykowski, Analytical model of the multi-service cellular network servicing multicast connections. Telecommun. Syst. 52(2), 1091-1100 (2013)

34. F Kelly, Reversibility and Stochastic Networks. (John Wiley \& Sons, Ltd., Chichester, West Sussex, 1979)

35. T Bonald, JW Roberts, Internet and the Erlang formula. Comput. Commun Rev. 42(1), 23-30 (2012)

36. VG Vassilakis, ID Moscholios, MD Logothetis, Call-level performance modelling of elastic and adaptive service-classes with finite population. IEICE Trans. 91-B(1), 151-163 (2008)

37. F Kelly, Notes on effective bandwidths. University of Cambridge. Technical report (1996)

38. JW Roberts (ed.), Performance Evaluation and Design of Multiservice Networks, Final Report COST 224 (Commission of the European Communities, Brussels, 1992), pp. 36-44

39. JW Roberts, V Mocci, I Virtamo (eds.), Broadband Network Teletraffic, Final Report of Action COST 242 (Commission of the European Communities, Springer, Berlin, 1996)

40. A Laiho, A Wacker, T Novosad (eds.), Radio Network Planning and Optimization for UMTS (John Wiley \& Sons, Ltd., Chichester, West Sussex, 2006)

41. S Faruque, Cellular Mobile Systems Engineering. (Artech House, London, 1997)

42. FP Kelly, Loss networks. Ann. Appl. Probab. 1(3), 319-378 (1991)

43. J Tyszer, Object-Oriented Computer Simulation of Discrete-Event Systems. (Kluwer Academic Publishers, Norwell, MA, USA, 1999)

44. M Głąbowski, M Stasiak, A Wiśniewski, P Zwierzykowski, Blocking probability calculation for cellular systems with WCDMA radio interface servicing PCT1 and PCT2 multirate traffic. IEICE Trans. Commun. E92-B(04), 1156-1165 (2009)

45. M Stasiak, A Wiśniewski, P Zwierzykowski, in Advanced Industrial Conference on Telecommunications/Service Assurance with Partial and Intermittent Resources Conference/E-Learning on Telecommunications Workshop. Uplink blocking probability for a cell with WCDMA radio interface and differently loaded neighbouring cells, vol. 0, (2005), pp. 402-407. doi:10.1109/AICT.2005.92

46. M Stasiak, P Zwierzykowski, in 9th Mediterranean Electrotechnical Conference. Analytical model of ATM node with multicast switching, vol. 2 (Tel-Aviv, 1998), pp. 683-6872

47. M Stasiak, P Zwierzykowski, Point-to-group blocking in the switching networks with unicast and multicast switching. Perform. Eval. 48(1-4), 247-265 (2002)

48. M Głąbowski, M Stasiak, P Zwierzykowski, Modelling of virtual-circuit switching nodes with multicast connections. Eur. Trans. Telecommun. 20(2), 123-137 (2009)
49. M Stasiak, P Zwierzykowski, D Parniewicz, in IEEE Global Telecommunications Conference. Modelling of the WCDMA interface in the UMTS network with soft handoff mechanism (Honolulu, HI, 2009), pp. 1-6

50. D Parniewicz, M Stasiak, P Zwierzykowski, Traffic engineering for multicast connections in multiservice cellular networks. IEEE Trans. Ind. Inform. 9(1), 262-270 (2013)

\section{Submit your manuscript to a SpringerOpen ${ }^{\mathcal{O}}$ journal and benefit from:}

- Convenient online submission

- Rigorous peer review

- Immediate publication on acceptance

- Open access: articles freely available online

- High visibility within the field

- Retaining the copyright to your article

Submit your next manuscript at $>$ springeropen.com 